\title{
Design of an advanced plasmonic sensor (consisting of a quadrilateral cavity, three rings with different dimensions and two waveguides) using refractive index change
}

\author{
Hamid Abbasi \\ University of Mazandaran,Iran.
}

\begin{abstract}
Using two metal insulated metal waveguides (MIM) and five resonator (a quadrilateral cavity and three rings with different dimensions), we design a plasmonic refractive index sensor. Using the time domain finite difference method, we examine and analyze the sensor performance (numerical analysis) and using the transmission line model method, we theoretically examine the performance of the sensor By summarizing these two methods and calculating the sensitivity coefficient $S$, quality coefficient $Q$ and figure of merit (FOM), a plasmonic sensor with good performance is obtained. Using the supermods of the resonators, we will achieve a stable wavelength spectrum and a suitable and stable sensitivity coefficient spectrum. Challenges and changes that occur in different modes of this sensor will lead to a balanced and relatively good structure. This designed sensor can be useful in the development of integrated optical circuits.
\end{abstract}

\section{Introduction}

Due to the ability to modulate light at the nanoscale and break the diffraction limit, polariton surface plasmons have received much attention. SP waves are a longitudinal charge density distribution that occurs when light is emitted in the plasmonic structure at the metal-dielectric interface. When plasmon is excited at this common surface, the fields are strongly enclosed at this surface and decompose rapidly on both sides of the metal and dielectric. Both metal and dielectric specimens can have complex refractive indices of $\varepsilon_{m}=\sqrt{n_{m}}$ and $\varepsilon_{d}=\sqrt{n_{d}}$, respectively. In electrodynamic structures, the surface plasmon will propagate as a transverse magnetic polarization mode (TM or P). The first condition for SP excitation is the polarization mode (TM) to produce the charge distribution on the metal surface. The SP phenomenon is easily understood and its main features can be determined by solving the Maxwell equation in the boundary value problem [1,2]. One of the most important tools that can use SP waves to develop integrated optical circuits is the plasmonic sensor. Plasmonic sensors are more suitable for integration due to their very small size and show stronger sensing performance [3]. Recently, various types of plasmonic sensors have appeared, such refractive index sensors [4], phase sensors [5], temperature sensors [6] and gas sensors [7]. The metal-insulator-metal (MIM) waveguide is one of the basic plasmonic waveguides with the capability to confine light within considerable propagating length [8] and many works of sensor are based on this structure [9-17]. In order for the sensor to offer excellent performance with high optical resolution, it is necessary to consider factors such as sensitivity, high transmission efficiency, optical stability, high resolution, high quality factor and FOM in the structure of plasmonic sensors. In this study, we analyze and simulate arrays of plasmonic waveguides and resonators so that we can design our desired plasmonic sensor. The purpose of this work is to achieve the desired parameters in plasmonic sensors and its development in integrated optical circuits [18].

\section{Structural model and theory analysis}

The schematic and two-dimensional view of the proposed plasmonic refractive index sensor is shown in Fig.1, which consists of a quadrilateral cavity resonator and three rings with different dimensions with two input and output waveguides. The structure of the sensor has two plasmonic waveguides with height $\mathrm{w} 1=50 \mathrm{~nm}$ and a quadrilateral cavity with height $\mathrm{w}_{2}=270$ $\mathrm{nm}$ and length $\mathrm{L}_{1}=200 \mathrm{~nm}$ and three rings. The upper ring at the top of the cavity has an inner 
radius of $r_{1}=62 \mathrm{~nm}$ and an outer radius of $R_{1}=92 \mathrm{~nm}$. The two nested rings at the bottom have an inner radius of $r_{2}=100 \mathrm{~nm}$ and $r_{3}=62 \mathrm{~nm}$ and an outer radius of $R_{2}=149 \mathrm{~nm}$ and $R_{3}$ $=62 \mathrm{~nm}$, respectively. Two pins, $\mathrm{P}_{\text {in }}$ and $\mathrm{P}_{\text {out }}$, are used to measure the input and output wax. The transfer rate is calculated by the following equation:

$\mathrm{T}=\mathrm{P}_{\text {out }} / \mathrm{P}_{\text {in }}$

(1)

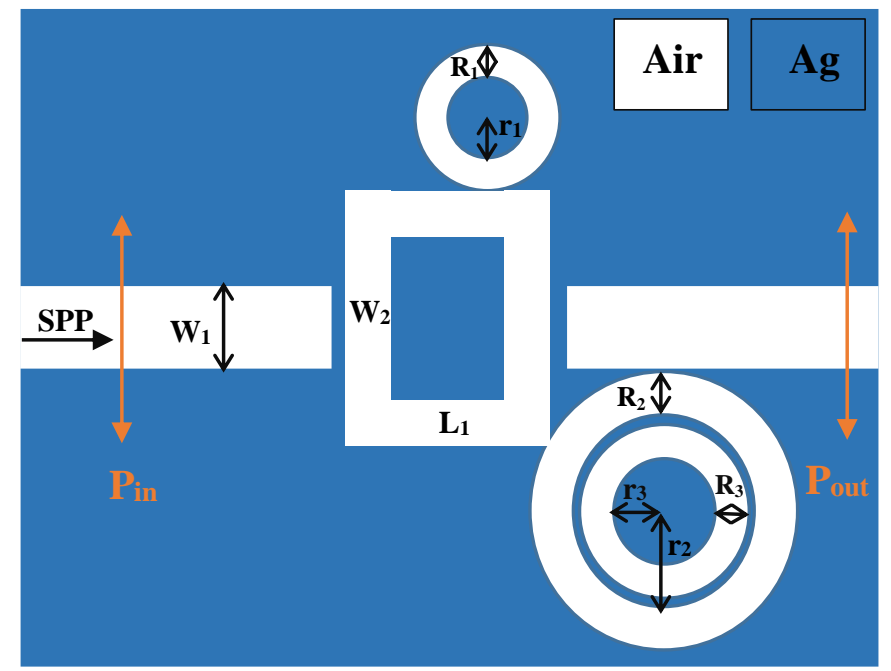

Fig.1. Schematic and two-dimensional image of a plasmonic sensor.

\section{Limited difference time domain method and transmission line model}

Using the time domain finite difference method, we examine and analyze the sensor performance (numerical analysis) and using the transmission line model method, we theoretically examine the performance of the sensor. Summarizing these two methods, we analyze the proposed structure resonance behavior and get a sensor with good performance is obtained [19-21]. The mesh in the $\mathrm{x}$ and $\mathrm{y}$ directions of the designed structure is $8 \mathrm{~nm}$. Many designed plasmonic devices use two-dimensional simulation to improve device performance and reduce simulation time. We also use the greeting model to show the optical properties of metals in simulation. The biggest advantage of the Hello model is that it can be incorporated into Maxwell equations in time-based numerical solutions (such as finite-time finite difference method):

$\varepsilon(\omega)=\varepsilon_{\infty}-\omega_{\mathrm{p}}^{2} / \omega^{2}+\mathrm{i \gamma} \omega$

Here $\omega_{\mathrm{p}}=1.37 \times 1016$ refers to bulk frequency for plasma, $\gamma=3.21 \times 1013$ means damping frequency for electron oscillation, $\varepsilon_{\infty}=1$ gives the medium constant for the infinite frequency, and $\omega$ shows incident light angular frequency. The TM wave starts moving from the left and is used for SPP excited waves and is published in the waveguideand the closer it gets to the output port, the less intense it becomes.

Each resonator reflects a portion of the input signal. As shown in Fig.2, the intermediate cavity absorbs the largest amount of TM wave and also has the highest field exchange with waveguides, thus having a greater impact on sensor performance. 


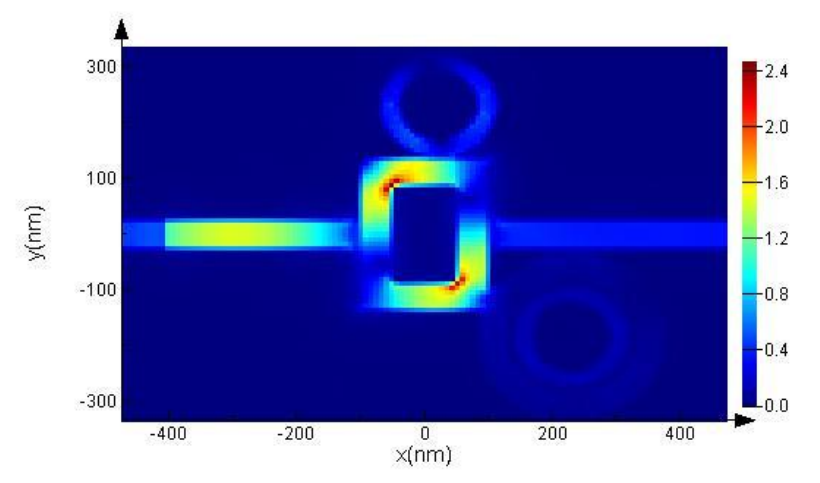

Fig.2. Electric field distribution at resonant frequency

If the field distribution in the sensor structure is similar, the energy loss is reduced. Therefore, in order to achieve the maximum field distribution in the desired structure, all dimensions must be optimized. Draw the structure transfer spectrum (Fig.3). First, we draw the transmission spectrum for a quadrilateral cavity. In the next step, we add the upper ring $\left(\mathrm{R}_{1}\right)$ and then we add the bottom ring $\left(R_{2}\right)$ and in the last step, we add the bottom nested ring $\left(\mathrm{R}_{3}\right)$.
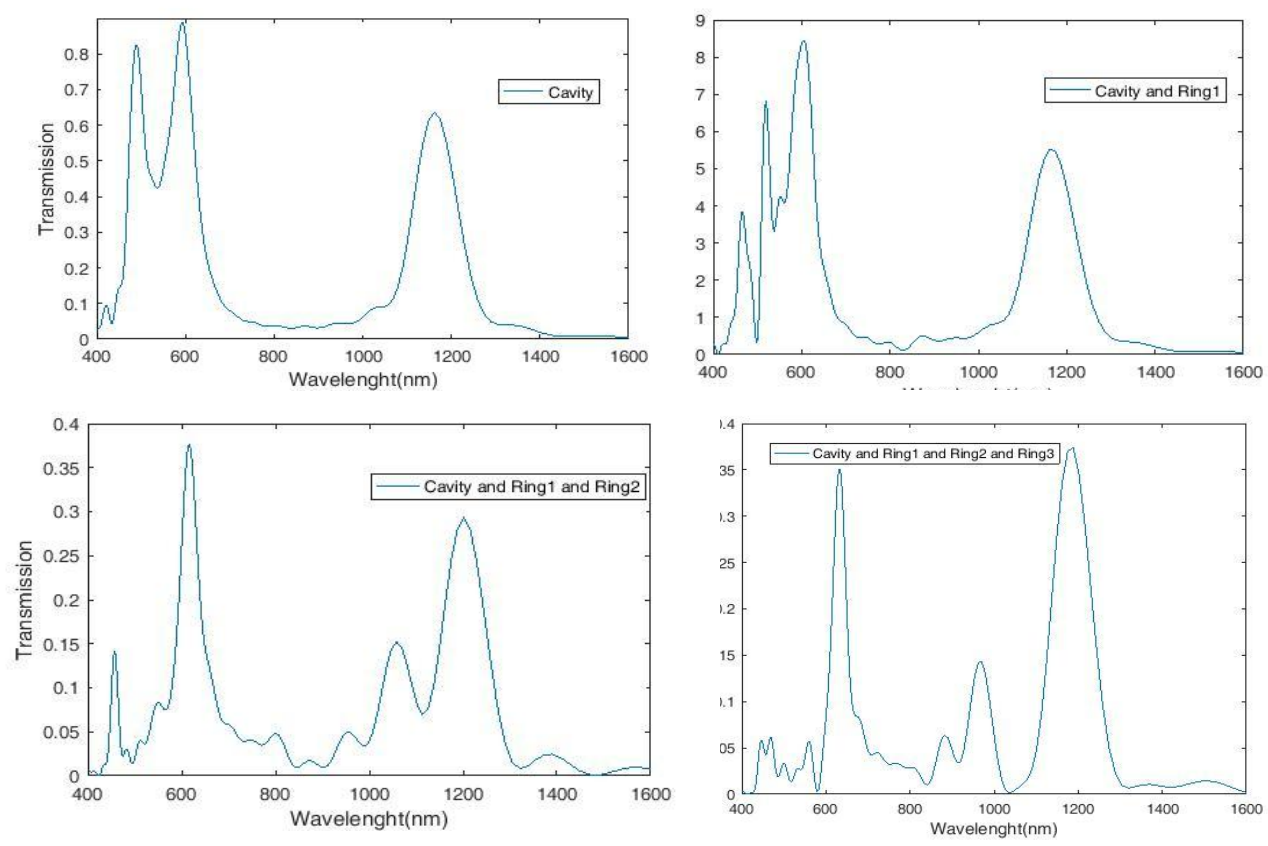

Fig.3. Sensor wave transmission spectra with Cavity and three rings $R_{1}$ and $R_{2}$ and $R_{3}$

The most suitable structure in the transmission spectra is formed when the cavity and the three rings are present at the same time. We now increase the dielectric refractive index by $0.01 \mathrm{~nm}$ from 1.11 to 1.2 , which changes the spectra and resonant wavelengths. The wave transmission spectrum obtained from the refractive index change is shown in Fig.4. 


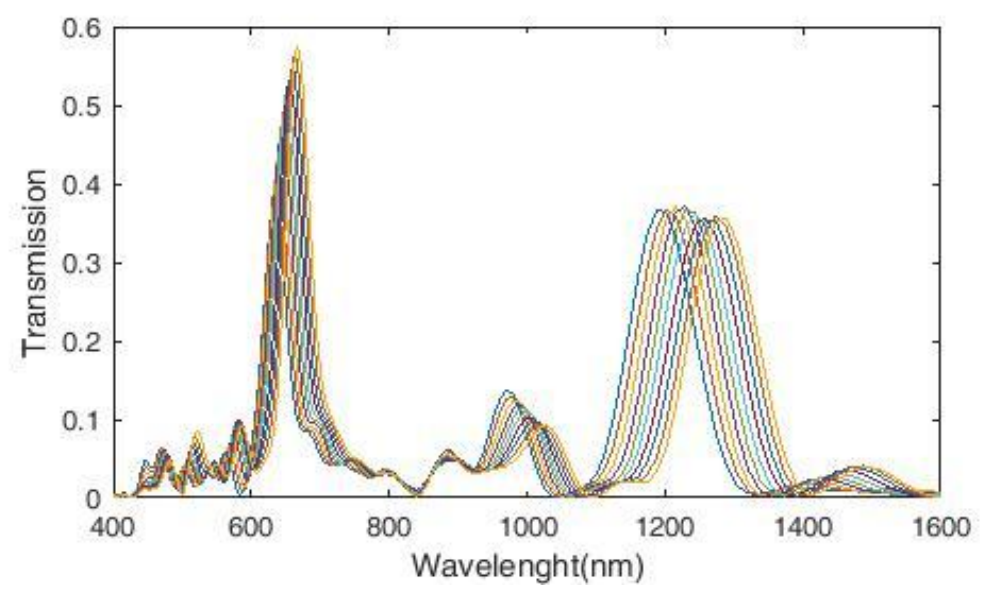

Fig.4. Designed refractive index sensor wavelength spectra.

To measure the performance of a plasmonic sensor, we need to consider several criteria. Sensitivity $\mathrm{S}$ is the first criterion and will be described as the change in resonant wavelength when the dielectric changes unit:

$\mathrm{S}=\Delta \lambda / \Delta \mathrm{n}(\mathrm{nm} / \mathrm{RIU})$

In this equation, $\Delta \mathrm{n}$ is the refractive index change and $\Delta \lambda$ is the resonance wavelength change. The sensor sensitivity coefficient diagram is shown in Figure 5. According to the figure, the maximum sensitivity for the refractive index is $\mathrm{n}=1.19$ (in mode2), which is equal to $1510 \mathrm{~nm}$ / RIU. According to this diagram, there is a relatively linear relationship between the refractive index and the resonant wavelength.

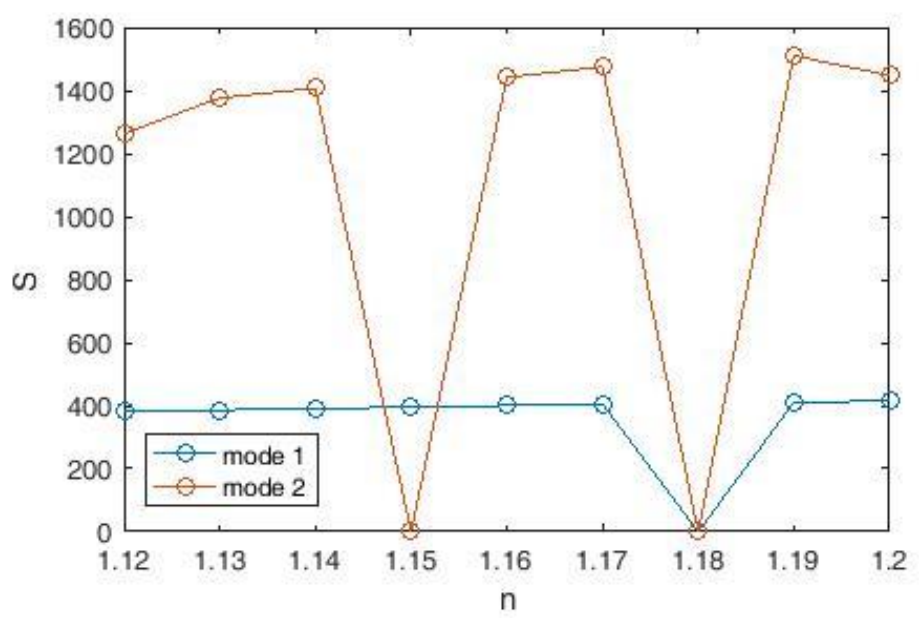

Fig.5. Plasmonic sensor sensitivity coefficient diagram

the figure of merit (FOM) is the second criterion whose equation is as follows:

$\mathrm{FOM}=\mathrm{S} / \mathrm{FWHM}$

(4)

The figure of merit (FOM) for the plasmonic sensor is plotted in Fig.6. According to the figure, the maximum figure of merit (FOM) for the refractive index is $n=1.19$ (in mode2), which is equal to $13.617 \mathrm{~nm} / \mathrm{RIU}$. 


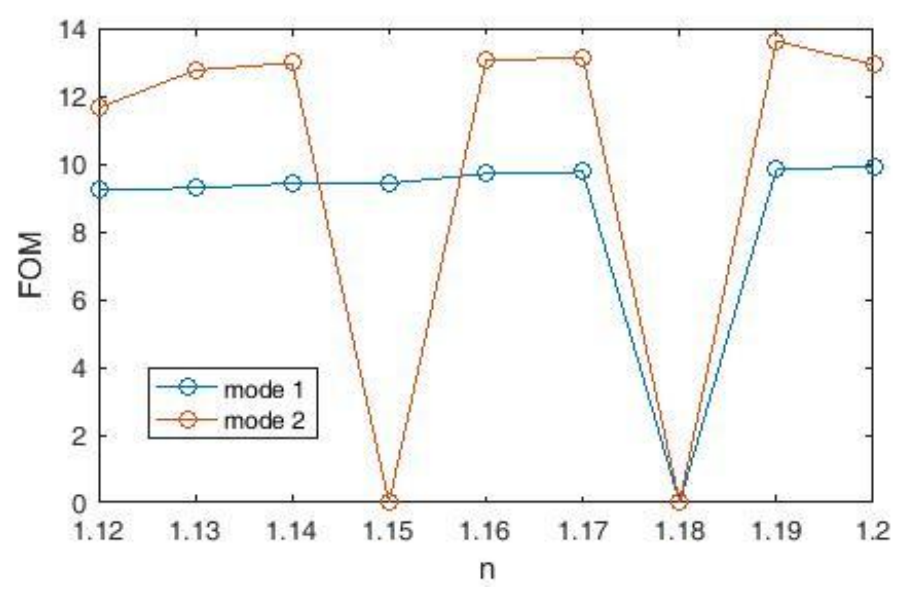

Fig.6. sensor figure of merit (FOM)

The last criterion is the quality factor Q, which is obtained according to Equation 5:

$\mathrm{Q}=\lambda$ res / FWHM

We see the diagram of the quality coefficient of the plasmonic sensor in Fig.7. According to the figure, the highest quality factor $\mathrm{Q}$ is for the refractive index $\mathrm{n}=1.19$ (in mode1), which is equal to $15.87 \mathrm{~nm} / \mathrm{RIU}$.

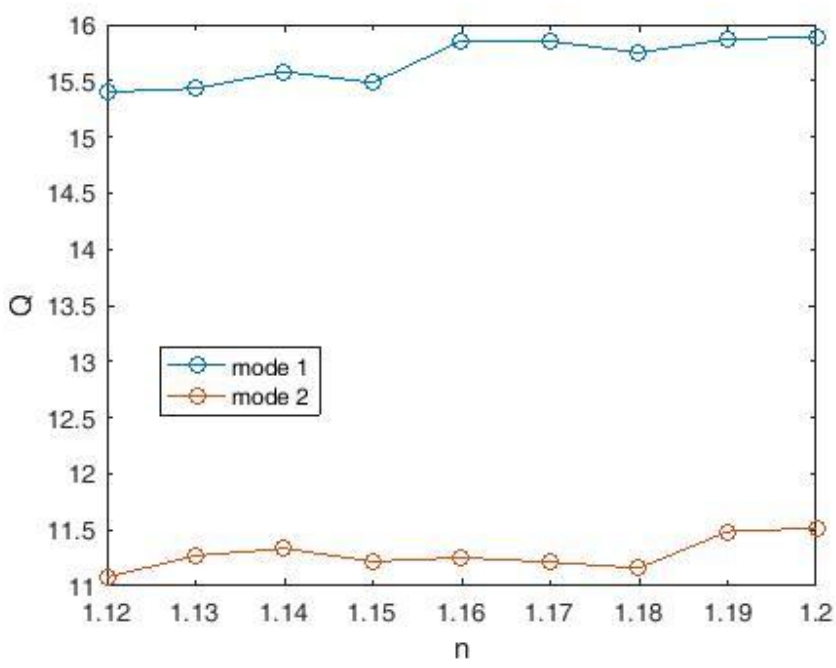

Fig.7. sensor quality coefficient diagram

Equations 3, 4 and 5 have evaluated the sensing capabilities of plasmon sensors and we conclude that this sensor has an optimal structure and has a high sensitivity.

\section{Conclusion}

An resonator system connected to a plasmonic waveguide, consisting of a metal-insulated metal waveguide (MIM) with a cavity and three rings, has been designed and numerically evaluated using the time-difference method.The two resonance peaks have different dependencies on the structural parameters due to their different mechanisms. So they will be easily adjustable. Due to its high resolution resolution, this sensor can detect a change in refractive index of 0.01 for materials with a refractive index of between 1.11 and 1.2. The maximum refractive index sensitivity of the proposed sensor structure is $1510 \mathrm{~nm} / \mathrm{RIU}$. The proposed structure is expected to provide guidelines for the design of nano-sensors. 


\section{Reference}

[1]. Sabine Szunerits, Rabah Boukherroub. Introduction to Plasmonics: Advancesand Applications. 2015.

[2]. Ebbesen, Thomas W, A. Dereux, and C. W. Barnes. Surface plasmon subwavelength optics. Nature 2003, 424.6950, 824-830.

[3]. Tong, L. Recent advances in plasmonic sensors. Sensors 2014, 14.5, 7959.

[4]. Y. Shen, J. H. Zhou, T. R. Liu, Y. T. Tao, R. B. Jiang, M. X. Liu, G. Xiao, J. Zhu, Z. K. Zhou, X. Wang, C. Jin, and J. Wang, Plasmonic gold mushroom arrays with refractive index sensing figures of merit approaching the theoretical limit. Nat. Commun 2013, 4, 2381.

[5]. Maisonneuve, M. Phase sensitive sensor on plasmonic nanograting structures. Optics Express 2011, 19.27, 26318-24.

[6]. Srivastava, Triranjita, R. Das, and R. Jha. Highly Sensitive Plasmonic Temperature Sensor Based on Photonic Crystal Surface Plasmon Waveguide. Plasmonics 2013, 8.2, 515-521.

[7]. Elsayed, Mohamed Y., Y. Ismail, and M. A. Swillam. Semiconductor plasmonic gas sensor using on-chip infrared spectroscopy. Applied Physics 2017, A 123.1, 113.

[8]. Maier, Stefan A. Plasmonics: Fundamentals and Applications. Springer Berlin 2014, 52.11, 49-74.

[9]. Shu, Changgan. The sensing characteristics of plasmonic waveguide with a ring resonator. Optics Express 2014, 22.7, 7669-77.

[10]. Yan, Shu Bin. A Refractive Index Sensor Based on a Metal-Insulator-Metal Waveguide-Coupled Ring Resonator. Sensors 2015, 15.11, 29183-91.

[11]. Huang, Ye Xiong. A plasmonic refractive index sensor based on a MIM waveguide with a sidecoupled nanodisk resonator. IEEE, International Conference on Embedded and Real-Time Computing Systems and Applications IEEE 2014, 1-5.

[12]. Wu, Tiesheng. A nanometeric temperature sensor based on plasmonic waveguide with an ethanolsealed rectangular cavity. Optics Communications 2015, 339, 1-6.

[13]. Tang, Y. Refractive Index Sensor Based on Fano Resonances in Metal-Insulator-Metal Waveguides Coupled with Resonators. Sensors 2017, 17.4, 784.

[14]. Zhang, Zhidong. Fano Resonance Based on Metal-Insulator-Metal Waveguide-Coupled Double Rectangular Cavities for Plasmonic Nanosensors. Sensors 2016, 16.5, 642.

[15]. Zafar, Rukhsar, and M. Salim. Enhanced Figure of Merit in Fano Resonance-Based Plasmonic Refractive Index Sensor. IEEE Sensors Journal 2015, 15.11, 6313-6317.

[16]. Chen, Jianjun. Coupled-Resonator-Induced Fano Resonances for Plasmonic Sensing with UltraHigh Figure of Merits. Plasmonics 2013, 8.4, 1627-1631.

[17]. Zhao, X., Z. Zhang, and S. Yan. Tunable Fano Resonance in Asymmetric MIM Waveguide Structure. Sensors 2017, 17, 7.

[18]. Zhaojian Zhang, Junbo Yang, Xin He, Jingjing Zhang, Jie Huang, Dingbo Chen, Yunxin Han. Plasmonic Refractive Index Sensor with High Figure of Merit Based on Concentric-Rings Resonator Sensors (Basel) 2018 Jan; 18(1): 116.

[19]. Allen Taflove; Susan C Hagness. Computational Electrodynamics: The Finite-difference Time-domain Method.2005.

[20]. J. Gierak, A. Madouri, A. L. Biance, E. Bourhis, G. Patriarche, C. Ulysse, D. Lucot, X. Lafosse, L. Auvray, L. Bruchhaus, and R. Gede "Sub-5 nm FIB direct patterning of nano devices," Microelectron. Eng. Vol. 84, pp. 779-783, 2007.

[21]. W. Wu, J. Yang, J. Zhang, J. Huang, D. Chen, and H. Wang, "Ultra high resolution filter and optical field modulator based on a surface plasmon polariton," Opt. Lett. Vol. 41, pp. 2310-2313, 2016. 